\title{
Improving the Quality and Electrical Properties of Poly(3,4-ethylenedioxythiophene):Poly(styrenesulfonate) (PEDOT:PSS) Film by Heating and Stirring the Solution
}

\author{
Juin J. Liou, ${ }^{1}$ Po-Hsun Hsu, ${ }^{2}$ Wen-Ray Chen, ${ }^{3 *}$ Dei-Wei Chou, ${ }^{4}$ Kuan-Wei Lee, ${ }^{5}$ \\ Na-Fu Wang, ${ }^{6}$ Shih-Wei Feng, ${ }^{2}$ and Chien-Jung Huang ${ }^{2 * *}$ \\ ${ }^{1}$ School of Information Engineering, Zhengzhou University, \\ \#100, Science Avenue, Zhengzhou, Henan Province, China \\ ${ }^{2}$ Department of Applied Physics, National University of Kaohsiung, \\ \#700, Kaohsiung University Rd., Nanzih Dist., Kaohsiung, Taiwan \\ ${ }^{3}$ Department of Electronic Engineering, National Formosa University, \#64, Wunhua Rd., Huwei, Yunlin, Taiwan \\ ${ }^{4}$ Department of Aviation \& Communication Electronics, Air Force Institute of Technology, \\ \#198, Jieshou W. Rd., Gangshan, Kaohsiung, Taiwan \\ ${ }^{5}$ Department of Electronic Engineering, I-Shou University, \\ \#1, Sec. 1, Syuecheng Rd., Dashu Dist., Kaohsiung, Taiwan \\ ${ }^{6}$ Department of Electronic Engineering, Center for Environmental Toxin and Emerging-Contaminant Research, \\ Super Micro Mass Research \& Technology Center, Cheng Shiu University, \\ \#840, Chengcing Rd., Niaosong Dist., Kaohsiung, Taiwan
}

(Received August 23, 2018; accepted November 26, 2018)

Keywords: PEDOT:PSS, heating and stirring, film hardness, closer structure

The improvement of the quality and electrical properties of a film will contribute to the stability and performance of an electronic product during component manufacturing. However, the pristine solution concentration ratio of poly(3,4-ethylenedioxythiophene):poly(styrenesulfonate) (PEDOT:PSS) to water is in the range of only $1.0-1.3 \mathrm{wt} \%$. Therefore, the pristine conductivity of PEDOT:PSS is poor because the conductive PEDOT is dispersed in excess water, resulting in the limitation of carrier transmission. In this study, a simple method of removing excess water in PEDOT:PSS solution by heating and stirring is proposed. The heating and stirring of the PEDOT:PSS solution can not only change the structure but also increase the conductivity of the PEDOT:PSS film. It is an effective method of improving the quality and electrical properties of the film simultaneously. Moreover, the fabrication process for the PEDOT:PSS film in this study is different from that of the commonly used multilayer film. A single film of high quality can be obtained in a short process time by heating and stirring. A high-quality single film is better than a multilayer film.

\section{Introduction}

Organic electronics or plastic electronics such as organic light-emitting diodes (OLEDs), liquid crystal displays (LCDs), touch panel displays, and wearable devices are currently attracting much interest as promising technologies. ${ }^{(1-5)}$ These devices all contain a transparent 
conductive film, which affects their operation. The quality and characteristics of transparent conductive films are important factors that determine the performance and application of consumer electronics. In particular, flexibility of electrodes is necessary for wearable devices and curved screens that will commonly be used in the future. However, the high cost and brittleness of indium tin oxide (ITO) generally used as the transparent electrode material limit the advantages of consumer electronics, resulting in increased demand for an alternative to ITO. ${ }^{(6,7)}$

Currently, the materials commonly used in the transparent conductive films that are expected to replace ITO are carbon nanotubes, graphene, thin metals, and conducting polymers. ${ }^{(8-11)}$ Among them, the conducting polymers have special properties that are interesting for new technologies. They not only have the electronic properties of semiconductors, but also the mechanical flexibility of plastic. Moreover, conjugated polymers are good materials for the fabrication of electronic devices. Their properties, such as disorder, can also be tuned within a certain band width by adjusting the external parameters during chemical synthesis. Among the conducting polymers, poly(3,4-ethylenedioxythiophene) (PEDOT) is promising as a nextgeneration transparent electrode material because its low cost, nontoxicity, easy processing, and mechanical flexibility can lead to cost-effective and flexible devices as well as roll-to-roll mass production. ${ }^{(12)}$

Although pure conductive PEDOT is difficult to process owing to its insolubility in water, it can still be dispersed in water by making use of the coulomb interaction with the counterion of the insulating poly(styrenesulfonate) (PSS). ${ }^{(13)}$ The concentration ratio of PEDOT:PSS solution to water is in the range of only $1.0-1.3 \mathrm{wt} \%$. Therefore, the conductivity of pristine PEDOT:PSS is poor because the conductive PEDOT is dispersed in excess water, resulting in the limitation of carrier transmission.

In this study, a simple method of removing the excess water in a PEDOT:PSS solution by heating and stirring is proposed. Heating and stirring not only changes the structure but also increases the conductivity of PEDOT:PSS solution. It is an effective method of improving the quality and electrical properties of a film simultaneously. It will contribute to better stability and performance during component manufacturing. The effect of heating and stirring on film quality and performance will be discussed in detail in this article.

\section{Experimental Procedure}

Glass substrates (H384 UR-GLASS-0.7MM, $0.7 \mathrm{~mm}$ ) with an area of $2.0 \times 2.0 \mathrm{~cm}^{2}$ were purchased from Flamegold Material Co. The glass substrates were cleaned with acetone, methanol, and deionized (DI) water successively for $10 \mathrm{~min}$ with each cleaner, and then dried in an oven after blowing with $\mathrm{N}_{2}$ gas. The cleaned glass substrates were treated with UV/ozone at $30 \mathrm{~W}$ for $60 \mathrm{~s}$ before spin-coating. The PEDOT:PSS aqueous solution used was Clevios PH1000 purchased from Heraeus Co. It has a PEDOT:PSS concentration in the range of 1.0-1.3 $\mathrm{wt} \%$, and the weight ratio of PSS to PEDOT is 2.5. The PEDOT:PSS aqueous solution was filtered through a syringe filter ( $0.45 \mathrm{~mm}$ pore size) before heating and stirring. The PEDOT:PSS solution was heated and stirred with a stirrer/hot plate (Corning PC-420D) to ensure homogeneous heat distribution. The PEDOT:PSS solutions were modified by heating at $100{ }^{\circ} \mathrm{C}$ 
and stirring at $200 \mathrm{rpm}$ in a sealed beaker (TAI FONG NO:107-25) between 5 and 24 min before further processing. Subsequently, the solution was spin-coated on a cleaned glass substrate at 1000 and $2000 \mathrm{rpm}$ for 10 and $20 \mathrm{~s}$, respectively. After that, the PEDOT:PSS film was baked at $130{ }^{\circ} \mathrm{C}$ for 20 min under ambient conditions in an oven.

The changes in the amount of the PEDOT:PSS solution were measured using a digital analytical balance (AND HR-200). The transmittances of the PEDOT:PSS films were measured using a UV/visible spectrometer (HITACHI, U-3900) at $550 \mathrm{~nm}$ wavelength. A surface microscopy image of the PEDOT:PSS film was measured by scanning electron microscopy (SEM, JEOL JSM-7001). The surface morphology, roughness, and adhesion map of the PEDOT:PSS films were measured by atomic force microscopy (AFM Bruker). The current image of the PEDOT:PSS film was measured by conductive atomic force microscopy (C-AFM Bruker). Moreover, for the chemical application of the PEDOT:PSS film was carried out X-ray photoelectron spectrometry (XPS, JEOL, JAMP-9500F).

\section{Results and Discussion}

\subsection{Effects of solution and film optical properties}

The concentration ratio of pure PEDOT:PSS solution to water is in the range of only 1.0-1.3 wt $\%$. Therefore, the conductivity of pristine PEDOT:PSS is poor because the conductive PEDOT is dispersed in excess water, resulting in the limitation of carrier transmission. Heating and stirring the PEDOT:PSS solution can remove the excess water in solution by thermal evaporation. It not only removes the water in the PEDOT:PSS solution but also makes the structures of PEDOT and PSS more similar to each other. A schematic illustration of the structural change is shown in Fig. 1.

The heating and stirring also affect the particle size of PEDOT:PSS (Fig. 2). This similar effect of heating temperature on particle size was reported by Friedel et al. ${ }^{(14)}$ The particle size of PEDOT:PSS decreased from 230 to $40 \mathrm{~nm}$ after heating from 60 to $90{ }^{\circ} \mathrm{C}$, representing

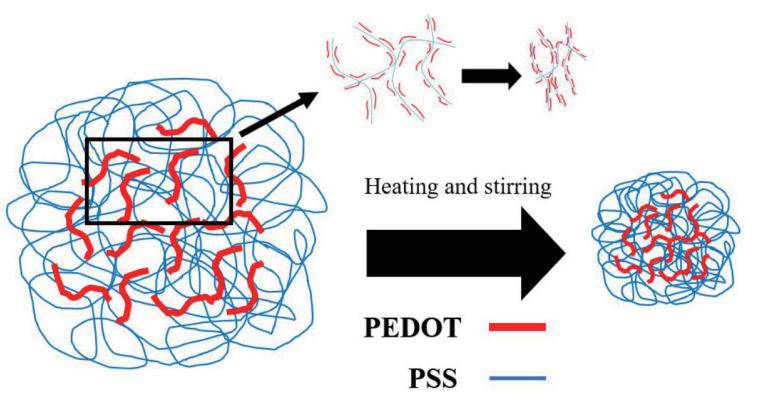

Fig. 1. (Color online) Schematic illustration of structural change caused by heating and stirring.

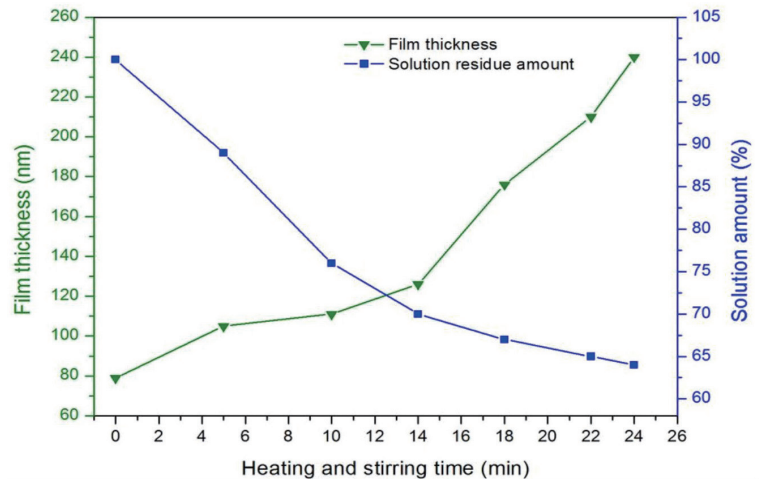

Fig. 2. (Color online) Variations of PEDOT:PSS film thickness and solution amount with time of heating and stirring at $100{ }^{\circ} \mathrm{C}$ and $200 \mathrm{rpm}$. 
a size reduction of $83 \%$. This allows the PEDOT:PSS particles to be more evenly dispersed in the film to promote charge transmission. The viscosity of the PEDOT:PSS solution was also increased owing to the evaporation of water in PEDOT:PSS induced by heating and stirring. The reduction in the evaporation rate of the PEDOT:PSS solution over time may be due to the saturation of the humidity in the sealed beaker. The relationship between the amount and concentration of the PEDOT:PSS solution with the time of heating and stirring at $100{ }^{\circ} \mathrm{C}$ and $200 \mathrm{rpm}$ is shown in Table 1.

Spin-coating at the same speeds of 1000 and $2000 \mathrm{rpm}$ for 10 and $20 \mathrm{~s}$ showed that the thickness of the PEDOT:PSS film depends on the time of heating and stirring. As the viscosity of the PEDOT:PSS solution increases, the thickness of the PEDOT:PSS film also increases because the high viscosity of the solution makes it difficult to separate it from the substrate by centrifugal force. The effects of heating and stirring on the optical properties of the PEDOT:PSS film were determined using a UV/visible spectrometer at $550 \mathrm{~nm}$ wavelength.

Figure 3 shows the variations of transmittance and solution amount with the time of heating and stirring. As the time of heating and stirring was increased, the transmittance of the PEDOT:PSS film decreased because the increase in the viscosity of the PEDOT:PSS solution leads to an increase in the thickness of the PEDOT:PSS film at a constant speed of the spincoating. The best parameters for optimizing the PEDOT:PSS solution are $65 \%$ of the solution amount after heating and stirring at $100{ }^{\circ} \mathrm{C}$ for $22 \mathrm{~min}$. An excessively long time of heating and stirring will deplete the water in the PEDOT:PSS solution, making it difficult to form an intact film of PEDOT:PSS by spin-coating owing to the agglomeration of the PEDOT:PSS solution. It will also result in a film transmittance of less than $80 \%$, which is undesirable in the application to transparent conductive electrodes in photovoltaic devices. Besides, longer immersion

Table 1

Amount and concentration of PEDOT:PSS solution with time of heating and stirring at $100{ }^{\circ} \mathrm{C}$ and $200 \mathrm{rpm}$.

\begin{tabular}{lrrrrrrr}
\hline Heating at $100{ }^{\circ} \mathrm{C}, 200 \mathrm{rpm}$ & $0 \mathrm{~min}$ & $5 \mathrm{~min}$ & $10 \mathrm{~min}$ & $14 \mathrm{~min}$ & $18 \mathrm{~min}$ & $22 \mathrm{~min}$ & $24 \mathrm{~min}$ \\
\hline Solution amount & $100 \%$ & $89 \%$ & $76 \%$ & $70 \%$ & $67 \%$ & $65 \%$ & $63.5 \%$ \\
Solution concentration & $100 \%$ & $112 \%$ & $131 \%$ & $142 \%$ & $150 \%$ & $153 \%$ & $157.0 \%$ \\
\hline
\end{tabular}

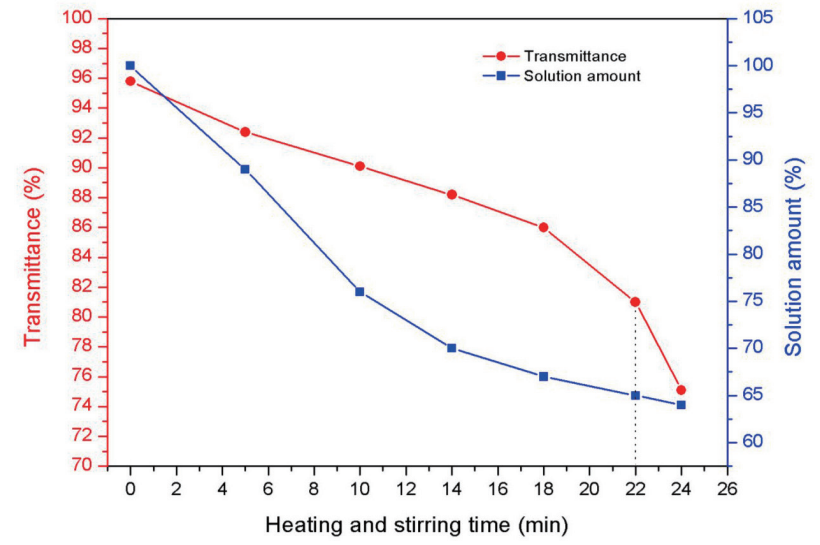

Fig. 3. (Color online) Variations of transmittance and solution amount with time of heating and stirring at $100{ }^{\circ} \mathrm{C}$ and $200 \mathrm{rpm}$. 
time will also cause more PEDOT to separate from PSS and change from coiled structures to elongated ones to scatter in the films, causing the transmittance of the films to be slightly lower because of the opaque PEDOT scattered in the films.

\subsection{Effects on film surface, morphology, and adhesion}

The effects of heating and stirring on the surface of a PEDOT:PSS film were observed by SEM. All the SEM images show a 2000-fold magnification. Figure 4 shows the top-view SEM images of PEDOT:PSS films after heating at $100{ }^{\circ} \mathrm{C}$ with different stirring times. White particles with 3\% sulfur atoms on the surface of the PEDOT:PSS films were observed using an energy-dispersive spectrometer (EDS). This suggests that the many aggregate particles of PEDOT:PSS in the PEDOT:PSS film cannot disperse uniformly and may form defects that will affect the performance during subsequent device production. Heating and stirring the PEDOT:PSS solution can decrease the particle size of PEDOT:PSS to induce uniform dispersal. The effect of heating and stirring on the uneven distribution of PEDOT:PSS particles is shown in Fig. 4(d). The white particles of PEDOT:PSS disappeared nearly completely after heating at $100{ }^{\circ} \mathrm{C}$ for $22 \mathrm{~min}$.

The effect of PEDOT:PSS particles with different heating temperatures in the same solution amount of $65 \%$ to improve the uniformity was also investigated in this study. The effect of optimizing the PEDOT:PSS solution by heating and stirring at 50,100, and $150{ }^{\circ} \mathrm{C}$ for different times is shown in Fig. 5. The top-view SEM images of the PEDOT:PSS film show the effects of heating and stirring at $100{ }^{\circ} \mathrm{C}$, which is different from those at other temperatures. Figure 5(a) shows the effect at a temperature of $50^{\circ} \mathrm{C}$. The PEDOT:PSS particles cannot be uniformly
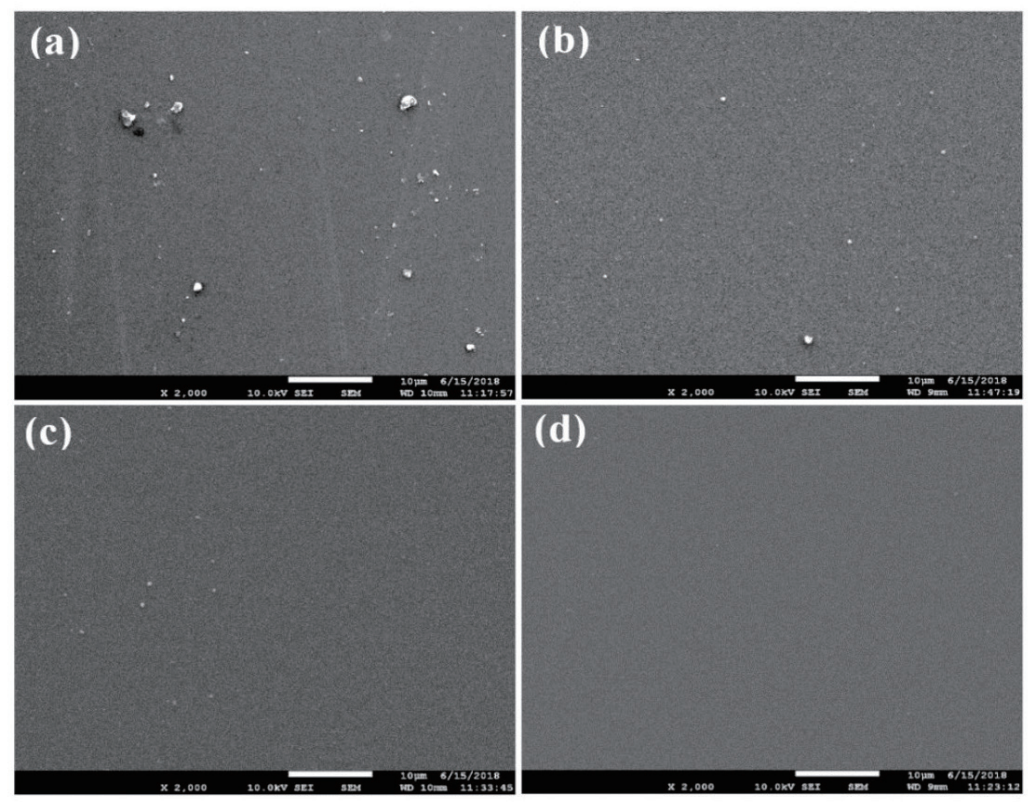

Fig. 4. Top-view SEM images of PEDOT:PSS films heated at $100{ }^{\circ} \mathrm{C}$ and stirred at $200 \mathrm{rpm}$ for different stirring times: (a) 0, (b) 10, (c) 18, and (d) $22 \mathrm{~min}$. Scale bar: $10 \mu \mathrm{m}$. 


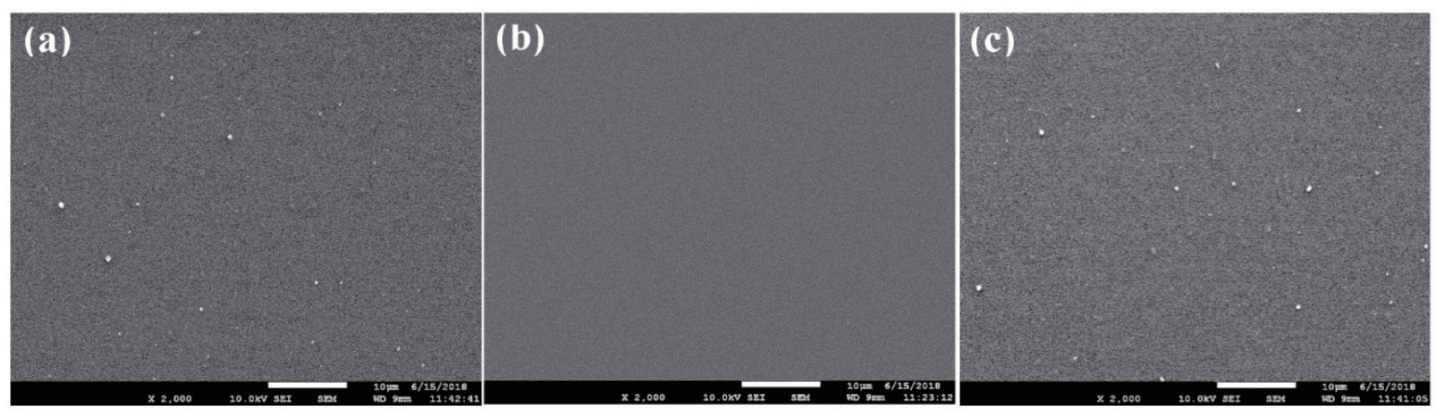

Fig. 5. Top-view SEM images of PEDOT:PSS films formed with $65 \%$ solution after stirring at $200 \mathrm{rpm}$ at different temperatures and times: (a) $50{ }^{\circ} \mathrm{C}, 100 \mathrm{~min}$, (b) $100{ }^{\circ} \mathrm{C}, 22 \mathrm{~min}$, and (c) $150{ }^{\circ} \mathrm{C}, 8.5 \mathrm{~min}$. Scale bar: $10 \mu \mathrm{m}$.

dissolved in the PEDOT:PSS solution owing to insufficient temperature, resulting in the formation of white particles on the surface of the PEDOT:PSS film. Figure 5(c) shows the effect at a temperature of $150{ }^{\circ} \mathrm{C}$. The excessively high heating temperature will evaporate the water in the PEDOT:PSS solution very rapidly, resulting in the white particles of PEDOT:PSS not having sufficient time to uniformly dissolve. In this study, the optimal parameter for optimizing the PEDOT:PSS solution at $100{ }^{\circ} \mathrm{C}$ was obtained. The effects of heating and stirring on the morphology variation in the PEDOT:PSS film were observed by AFM. All the images were of an area $1 \times 1 \mu \mathrm{m}^{2}$ in size.

The AFM images in Fig. 6 clearly show the changes in the PEDOT:PSS film morphology after heating at $100{ }^{\circ} \mathrm{C}$ and stirring at $200 \mathrm{rpm}$ for different stirring times. The PEDOT:PSS films were seen to be more aggregated in morphology after heating and stirring for longer periods, as shown in Fig. 6(d). The AFM images show that the PEDOT:PSS film surface roughness slightly increased after heating and stirring for longer periods with root mean square (RMS) roughness values of $1.21,1.54,1.68$, and $1.85 \mathrm{~nm}$. The heating and stirring to remove the water in the PEDOT:PSS solution will bring the PEDOT nearer to the film surface. ${ }^{(15)}$ The effects of heating and stirring on adhesion variation in the PEDOT:PSS film were observed in the AFM adhesion map. All the AFM images were of $1 \times 1 \mu \mathrm{m}^{2}$ size.

Figure 7 shows clear changes in the PEDOT:PSS film morphology in the adhesion map after heating at $100{ }^{\circ} \mathrm{C}$ and stirring at $200 \mathrm{rpm}$ for different stirring times. They were seen to be more compact and to darken in the adhesion map of the PEDOT:PSS films after heating and stirring for longer periods, as shown in Fig. 7(d). The adhesion of the PEDOT:PSS films was also decreased from 35 to $7 \mathrm{nN}$ after heating and stirring for $22 \mathrm{~min}$, representing an adhesion reduction of $80 \%$. The force curve measurements on the glass substrate showed little or no adhesion force. ${ }^{(16)}$ Thus, the reduction of adhesion in the PEDOT:PSS film can be used as evidence of the increase in film hardness.

\subsection{Effects of film conductivity and binding energy}

The effects of heating and stirring on the conductivity of the PEDOT:PSS film were observed from the c-AFM current images. All the AFM images were of areas $1 \times 1 \mu \mathrm{m}^{2}$ in 

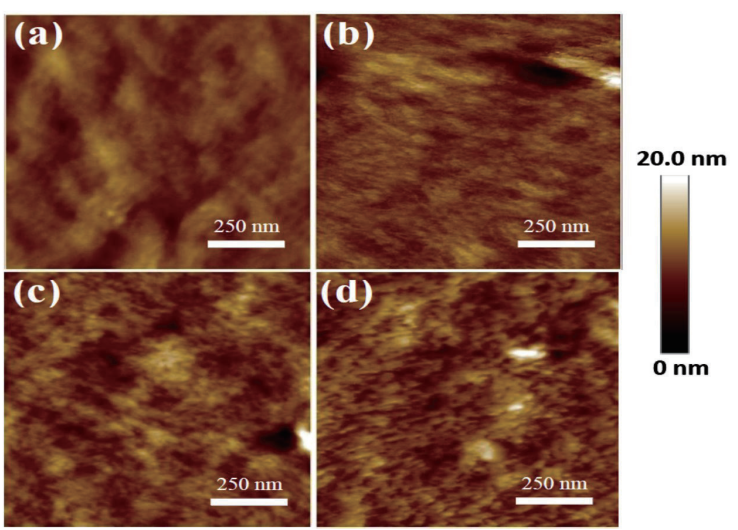

Fig. 6. (Color online) AFM topographic images of PEDOT:PSS films heated at $100{ }^{\circ} \mathrm{C}$, and stirred at 200 rpm for different stirring times: (a) 0 , (b) 10, (c) 18, and (d) $22 \mathrm{~min}$. All images are of $1 \times 1 \mu \mathrm{m}^{2}$ areas.

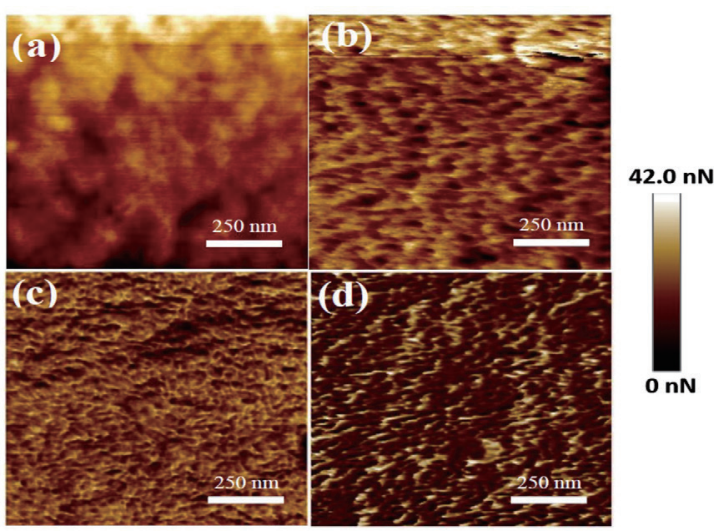

Fig. 7. (Color online) AFM adhesion map of PEDOT:PSS films heated at $100{ }^{\circ} \mathrm{C}$ and stirred at 200 rpm for different stirring times: (a) 0, (b) 10, (c) 18, and (d) $22 \mathrm{~min}$. All images are of $1 \times 1 \mu \mathrm{m}^{2}$ areas.

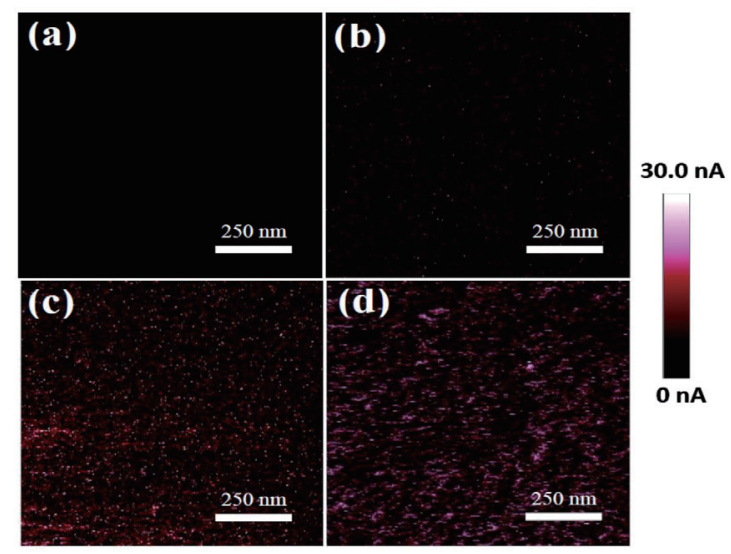

Fig. 8. (Color online) AFM current images of PEDOT:PSS films heated at $100{ }^{\circ} \mathrm{C}$ and stirred at $200 \mathrm{rpm}$ for different stirring times: (a) 0 , (b) 10 , (c) 18 , and (d) $22 \mathrm{~min}$. All images are of $1 \times 1 \mu \mathrm{m}^{2}$ areas.

size. Figure 8 shows that as the time of heating and stirring increases, there is a considerable increase in the total number of conductive pathways observed by c-AFM. The PEDOT:PSS films showed a rapid increase in the number of conductive hot spots after heating and stirring owing to the change in the PEDOT:PSS structure. The heating and stirring to remove the water in the PEDOT:PSS solution not only brings the PEDOT nearer to the film surface but also makes the structures of PEDOT and PSS more similar to each other. The PEDOT:PSS films showed an increase in current density from $0.52,4.10$, and 6.47 to $6.91 \mathrm{nA} / \mu \mathrm{m}^{2}$ after heating and stirring for longer periods, representing a 13 -fold increase. The RMS longitudinal conductivity of PEDOT:PSS films was also increased by 7 times from $0.96,1.27$, and 6.12 to $6.69 \mathrm{nA} / \mu \mathrm{m}^{2}$ after heating and stirring for longer periods. This suggests that the vertical and transversal connectivities between PEDOT grains are indeed improved, resulting in the increased bulk conductivity attributed to an average decrease in the intergrain distance and an increase in the number of interparticle connections. ${ }^{(17-19)}$ 


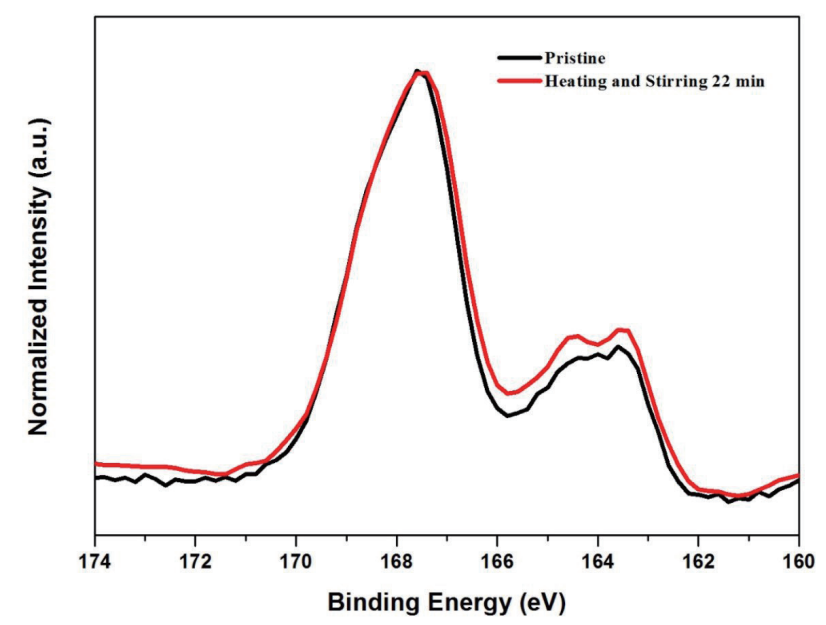

Fig. 9. (Color online) XPS spectra of pristine and solution-optimized PEDOT:PSS films.

The effects of heating and stirring on binding energy in the PEDOT:PSS film were measured by XPS, which is a surface-sensitive technique, but no lateral resolution is examined because the size of the analyzed spot is a few $\mathrm{mm}^{2}$. Both PEDOT and PSS contain one sulfur atom per repeat unit. The sulfur (S) atom in PEDOT is within the thiophene ring, whereas the $\mathrm{S}$ atom in PSS is included in the sulfonate moiety. Owing to those different chemical environments, the $\mathrm{S}(2 \mathrm{p})$ electrons of PEDOT and PSS have different binding energies so that the composition of PEDOT-PSS can be analyzed by XPS. The S (2p) peak at a binding energy between 167 and $171 \mathrm{eV}$ originates from the sulfur atoms in PSS, and the doublet peaks at 164.4 and 165.6 $\mathrm{eV}$ correspond to the sulfur signal of PEDOT, as shown in Fig. 9. ${ }^{(20)}$ Figure 9 shows the XPS spectra of pristine and solution-optimized PEDOT:PSS films. The binding energies of PEDOT in the PEDOT:PSS film after solution optimization by heating and stirring at $100{ }^{\circ} \mathrm{C}$ for $22 \mathrm{~min}$ slightly increased. The increase in binding energy in the conductive PEDOT measured under the same measurement range was attributed to a closer structure that allows them to become nearer to each other in the PEDOT:PSS film, which is beneficial to charge transmission.

\section{Conclusions}

A simple method of removing excess water in a PEDOT:PSS solution by heating and stirring has been proposed. The structure between the PEDOT and the PSS became more compact because heating and stirring the PEDOT:PSS solution will evaporate the water in the solution. The current density of the PEDOT:PSS film was increased from 0.52 to $6.91 \mathrm{nA} / \mu \mathrm{m}^{2}$, which is 13 times greater than that of a pristine film, after heating and stirring the PEDOT:PSS solution. It can not only increase the conductivity but also change the quality of the PEDOT:PSS film. The adhesion of the PEDOT:PSS film was decreased from 35 to $7 \mathrm{nN}$, representing an adhesion reduction of $80 \%$. In other words, the hardness of the film was increased. Heating and stirring is an effective method of improving the quality and electrical properties of the film simultaneously. Furthermore, the fabrication process for the PEDOT:PSS film is different from 
that of the commonly used multilayer film. A single film of high quality, which is better than a multilayer film, can be obtained in a short process time after heating and stirring. A simple process and high-quality technique to improve the PEDOT:PSS monolayer film by heating and stirring can improve the performance of devices after the subsequent manufacturing.

\section{Acknowledgments}

This work was partially supported by the Ministry of Science and Technology (MOST) of Taiwan under contract No MOST 106-2221-E-390-016.

\section{References}

1 C. J. Huang, T. H. Meen, K. C. Liao, and Y. K. Su: J. Phys. Chem. Solids 70 (2009) 765.

2 W. Kim, A. Mäkinen, N. Nikolov, R. Shashidhar, H. Kim, and Z. Kafafi: Appl. Phys. Lett. 80 (2002) 3844.

3 X. Crispin, F. Jakobsson, A. Crispin, P. Grim, P. Andersson, A. Volodin, C. Van Haesendonck, M. Van der Auweraer, W. R. Salaneck, and M. Berggren: Chem. Mater. 18 (2006) 4354.

4 G. Crawford: Flexible Flat Panel Displays (John Wiley \& Sons, New York, 2005).

5 W. Honda, S. Harada, T. Arie, S. Akita, and K. Takei: Adv. Funct. Mater. 24 (2014) 3299

6 C. S. Tao, J. Jiang, and M. Tao: Sol. Energy Mater. Sol. Cells 95 (2011) 3176.

7 A. Chipman: Nature 449 (2007) 7159.

8 J. van de Lagemaat, T. M. Barnes, G. Rumbles, S. E. Shaheen, T. J. Coutts, C. Weeks, I. Levitsky, J. Peltola, and P. Glatkowski: Appl. Phys. Lett. 88 (2006) 233503.

9 T.-H. Han, Y. Lee, M.-R. Choi, S.-H. Woo, S.-H. Bae, B. H. Hong, J.-H. Ahn, and T.-W. Lee: Nat. Photonics 6 (2012) 105.

10 H. Wu, D. Kong, Z. Ruan, P.-C. Hsu, S. Wang, Z. Yu, T. J. Carney, L. Hu, S. Fan, and Y. Cui: Nat. Nanotechnol. 8 (2013) 421.

11 M. Vosgueritchian, D. J. Lipomi, and Z. Bao: Adv. Funct. Mater. 22 (2012) 421.

12 F. C. Krebs: Org. Electron. 10 (2009) 761.

13 S. Kirchmeyer and K. Reuter: J. Mater. Chem. 15 (2005) 2077.

14 B. Friedel, T. J. Brenner, C. R. McNeill, U. Steiner, and N. C. Greenham: Org. Electron. 12 (2011) 1736.

15 L. S. Pingree, B. A. MacLeod, and D. S. Ginger: J. Phys. Chem. C 112 (2008) 7922.

16 A. D. Bershadsky, N. Q. Balaban, and B. Geiger: Annu. Rev. Cell Dev. Biol. 19 (2003) 677.

17 J. Huang, P. F. Miller, and J. S. Wilson: Adv. Funct. Mater. 15 (2005) 290.

18 G. Greczynski, T. H. Kugler, M. Keil, W. Osikowicz, M. Fahlman, and W. R. Salaneck: J. Electron. Spectrosc. Relat. Phenom. 121 (2001).

19 M. P. de Jong, L. J. van Ijzendoorn, and M. J. A. de Voigt: Appl. Phys. Lett. 77 (2000) 2255.

20 S.-I. Na, G. Wang, S.-S. Kim, T.-W. Kim, S.-H. Oh, B.-K. Yu, T. Lee, and D.-Y. Kim: J. Mater. Chem. 19 (2009) 9045 . 